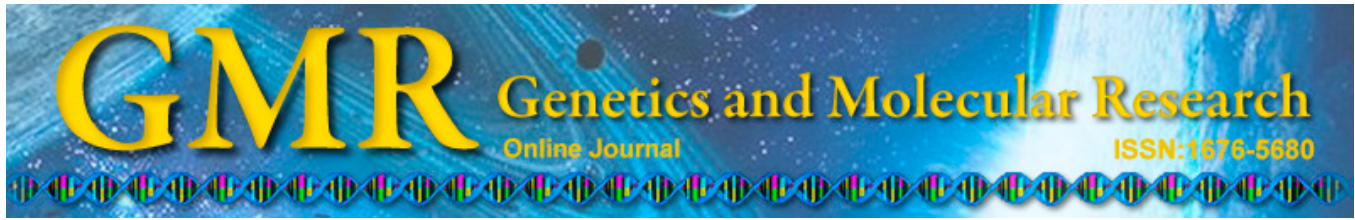

\title{
Increased human epididymis protein 4 in benign gynecological diseases complicated with chronic renal insufficiency patients
}

\author{
Y.W. Lv, L. Yang, M. Zhang, L.H. Jiang, J.H. Niu, J. Hou and X.H. Cui \\ Department of Obstetrics and Gynecology, PLA 463rd Hospital, Shenyang, \\ Liaoning Province, China \\ Corresponding author: Y.W. Lv \\ E-mail: yanwenlven@126.com
}

Genet. Mol. Res. 14 (1): 2156-2161 (2015)

Received June 27, 2014

Accepted February 10, 2015

Published March 27, 2015

DOI http://dx.doi.org/10.4238/2015.March.27.2

\begin{abstract}
We examined the serum concentration of human epididymis protein (HE4) in patients with benign gynecological diseases complicated with chronic renal deficiency and its significance in the differential diagnosis of benign and malignant gynecological diseases. Serum HE4 and cancer antigen 125 concentrations were detected by chemiluminescence. Clinically or pathologically confirmed gynecological diseases were grouped and retrospectively analyzed, including 50 cases of gynecological benign diseases, 35 cases of non-mucinous epithelial ovarian carcinoma, 36 cases of endometrial adenocarcinoma, 15 cases of gynecological benign diseases patients complicated with chronic renal deficiency, 15 cases of gynecological diseases without chronic renal deficiency, and 30 normal controls. Serum HE4 values in the ovarian cancer group, endometrial cancer group, gynecological benign diseases with chronic renal deficiency group, and chronic renal deficiency group were significantly increased compared with the benign gynecological diseases and normal control groups, showing a significant difference $(\mathrm{P}<0.001)$. A comparison of 4 groups with high HE4 showed that the HE4 level in the 2 groups
\end{abstract}


with renal deficiency were higher than those in the ovarian cancer and endometrial cancer groups, but the difference was not significant $(\mathrm{P}>$ $0.05)$; there was no significant difference between 2 groups with renal deficiency $(\mathrm{P}>0.05)$. Serum concentration of HE4 was high in patients with chronic renal deficiency, which should be distinguished during differential diagnosis of gynecological benign and malignant tumors in patients with chronic renal deficiency to avoid misdiagnosis.

Key words: Chronic renal deficiency; Differential diagnosis; Gynecological tumor; Human epididymis protein

\section{INTRODUCTION}

The most widely recognized ovarian cancer marker worldwide is cancer antigen 125 (CA-125), but its sensitivity and specificity are low (Moore et al., 2012b). Human epididymis protein 4 (HE4) is a recently identified tumor marker that has been increasingly used in gynecology (Hertlein et al., 2012; Moore et al., 2012a). A previous study found that in the differential diagnosis of ovarian epithelial cancer and benign ovarian tumor, the sensitivity of HE4 was higher than that of CA-125 (Hellström et al., 2003), and HE4 combined with CA125 was helpful for identifying benign and malignant ovarian tumors (Moore et al., 2008). HE4 is valuable for diagnosing endometrial cancer as well as for monitoring and evaluating the curative effects (Moore et al., 2011). Therefore, since 2011, our hospital has carried out HE4 detection for the differential diagnosis of ovarian masses and abnormal uterine bleeding to exclude endometrial cancer and ovarian cancer. Serum HE4 in patients with chronic renal deficiency has been observed to be significantly higher than in patients with benign gynecological diseases. Therefore, we analyzed the relationship between HE4 and chronic renal deficiency and gynecological benign and malignant diseases to improve the understanding of high serum HE4 in the differential diagnosis of benign and malignant disease of Department of Gynecology.

\section{MATERIAL AND METHODS}

\section{General materials}

From January 2011 to January 2014, the research subjects collected were patients of the 463rd Hospital of the People's Liberation Army, including 136 cases in the gynecological clinic, 15 cases with chronic renal deficiency in the Department of Internal Medicine, and 30 normal controls, including a total of 181 cases.

A retrospective analysis was performed to evaluate the gynecological outpatients and inpatients for pelvic masses and abnormal uterine bleeding. Serum levels of HE4 were detected to exclude ovarian cancer and endometrial cancer. The 136 cases of gynecological disease clinical or pathological diagnosis were divided into the gynecological benign diseases group (50 cases), non-mucinous epithelial ovarian cancer group (35 cases), endometrial adenocarcinoma group (36 cases), and benign gynecological diseases group accompanied with chronic renal deficiency (15 cases). All cases were premenopausal women; the average ages in all groups showed no statistically significant differences. All cases were excluded of lung cancer, breast cancer, colon cancer, and other cancer by computed tomography or ultrasound. 


\section{Diagnostic standard of chronic renal deficiency (CKD)}

Diagnostic standards were in accordance with American Kidney Foundation DOQI Expert Group on Chronic Renal Deficiency and glomerular filtration CRF staging.

\section{HE4 and CA-125 detection}

Detection was performed by chemiluminescence. Using the Fujirebio Diagnostics, Inc. Kit (Elof Lindalvs, Goteborg Sweden), the normal reference values were respectively 150 $\mathrm{pM}$ and $35 \mathrm{U} / \mathrm{mL}$.

\section{Statistical analysis}

The Statistical software SPSS version 13.0 was used for statistical analysis (SPSS, Inc., Chicago, IL, USA).

\section{RESULTS}

\section{Comparison results of HE4 group}

As shown in Table 1, serum HE4 values in the ovarian cancer, endometrial cancer, gynecological benign diseases with CKD, and CKD groups were significantly increased compared with in the benign gynecological diseases and normal control groups $(\mathrm{P}<0.001)$. In the comparison of the 4 groups with high HE4, the HE4 level for 2 groups with renal deficiency were higher than those in the ovarian cancer and endometrial cancer groups, but the difference was not significant $(\mathrm{P}>0.05)$; there was no significant difference between the 2 groups with renal deficiency $(\mathrm{P}>0.05)$. There was no significant difference in the comparison of gynecological benign disease and the normal control group $(\mathrm{P}>0.05)$.

\begin{tabular}{|c|c|c|}
\hline & HE4 (pM) & CA125 (35U/mL) \\
\hline Normal control $(\mathrm{N}=30)$ & $39.1(8.8-79.6)$ & $18.2(3.9-28.3)$ \\
\hline Gynecological benign diseases $(\mathrm{N}=50)$ & $43.6(33.6-109.0)$ & $59.9(8.4-133.2)$ \\
\hline Gynecological benign diseases +CKD $(\mathrm{N}=15)$ & $839.9(312.9-1864.5)$ & $66.2(5.1-149.2)$ \\
\hline $\mathrm{CKD}(\mathrm{N}=15)$ & $856.3(415.1-1208.9)$ & $12.5(6.7-23.8)$ \\
\hline Ovary cancer $(\mathrm{N}=35)$ & $589.9(190.6-875.3)$ & $263.5(88.6-378.3)$ \\
\hline Endometrial cancer $(\mathrm{N}=36)$ & $534.6(212.8-857.6)$ & $53.7(34.9-143.8)$ \\
\hline
\end{tabular}

\section{Comparison of CA-125 among groups}

As shown in Table 1, CA-125 in the ovarian cancer group increased significantly, showing a significant difference compared with other groups $(\mathrm{P}<0.01)$. Compared with the normal control group and CKD group, CA-125 in the endometrial cancer group, gynecological benign diseases group, and gynecological benign diseases with CKD group, values were significantly increased $(\mathrm{P}<0.05)$, but there was no significant difference among the endometrial cancer group, gynecological benign diseases group, and gynecological benign diseases 
with CKD group $(\mathrm{P}>0.05)$; the normal control and CKD group showed no significant difference $(\mathrm{P}>0.05)$.

\section{DISCUSSION}

\section{Current research on HE4}

Kirchhoff et al. (1991) first identified HE4 in human epididymis epithelial cells. HE4 is normally expressed in the respiratory tract, genital tract (such as female endocervical glands, endometrial glands, Bartholin's gland tubal, and organization), breast, renal distal tubules, colon mucosa, and salivary glands, and is highly expressed in lung cancer, breast cancer, transitional cell carcinoma, and pancreatic cancer (Galgano et al., 2006). Gynecological studies have found that ovarian epithelial cells do not express HE4. Another study found that different types of ovarian cancer express different levels of HE4, including $50 \%$ of ovarian serous carcinomas and $100 \%$ of ovarian endometrioid carcinoma, $93 \%$ of ovarian clear cell carcinoma, and is not expressed in ovarian mucinous carcinoma (Drapkin et al., 2005). HE4 is highly expressed in endometrial carcinoma and was found to be correlated with tumor stage (Bignotti et al., 2011). Therefore, an increasing number of studies have predicted HE4 to be an important tumor marker for early ovarian cancer and endometrial cancer, and can contribute to the curative effect of ovarian cancer, prediction of recurrence after operation, and prognosis evaluation of endometrial cancer (Hellström et al., 2003; Moore et al., 2008, 2011, Bignotti et al., 2011; Hertlein et al., 2012; Schummer et al., 2012). The results of this study are consistent with those of previously reported studies.

\section{Role of HE4 in the differential diagnosis of benign and malignant gynecological tumors}

Moore et al. (2012b) examined 1042 patients with gynecological benign diseases and found that HE4 and CA-125 levels were increased by 3 and $67 \%$, respectively, in the endometriosis group, 8 and $20 \%$ in ovarian serous tumors, 8 and $26 \%$ in the fibrous tumors, 10 and $37 \%$ in inflammation disease, 1 and $21 \%$ in dermoid cysts, and 8 and $29 \%$ in overall benign disease $(\mathrm{P}<0.001)$. Gynecological benign diseases rarely show increased HE4, but show higher levels of CA-125, particularly in premenopausal women (Holcomb et al., 2011; Moore et al., 2012a). Therefore, HE4 is more sensitive than CA-125 for the differential diagnosis of benign and malignant tumors in premenopausal women with adnexal masses, and single HE4 or combined HE4 with CA-125 is helpful for the differential diagnosis of benign and malignant ovarian tumors. Overall, ultrasound diagnosis of ovarian masses with increased HE4 and CA-125 suggests the presence of ovarian cancer; if only increased CA125 is observed, the patient may have ovarian endometriosis or other benign diseases; if only increased HE4 is observed, the patient may have ovarian or endometrial cancer or other malignant tumors. Consistent with results of Chinese and worldwide studies (Moore et al., 2011; Bandiera et al., 2011; Jacob et al., 2011; Lenhard et al., 2011; Montagnana et al., 2011; Van Gorp et al., 2011; Kadija et al., 2012), our retrospective analysis showed that HE4 can be used as a marker for the diagnosis of ovarian cancer and endometrial cancer, and is more effective than using CA-125. 


\section{Increased HE4 in CKD}

An important finding of this study was that serum HE4 was significantly increased in patients with gynecological benign diseases complicated with chronic renal deficiency, and even higher in patients with ovarian cancer and endometrial cancer. Nagy et al. (2012) first reported increased HE4 in 113 cases of chronic renal deficiency (Lindquist and Mertens, 2013). Their study was grouped according to the degree of renal deficiency, showing that HE4 in each renal deficiency group was statistically significantly higher than in the control group, and increased HE4 was related to damage degree and renal function, while CA-125 was only significantly increased in those with renal damage. There have been no studies examining these factors in China. Our study included a limited number of cases, and we did not perform further staging on the renal deficiency group. The study found that HE4 in the 2 groups of patients complicated by chronic renal deficiency was higher than in normal controls and the gynecological benign disease group with no renal deficiency, while increased CA-125 was not statistically significantly different compared with the corresponding control groups. The results suggest that in the differential diagnosis of gynecological benign and malignant tumors, high HE4 and renal deficiency should be analyzed to avoid misdiagnosis. Renal deficiency patients are often in a poor systemic state and economic condition, and benign tumors should be conservatively treated. If the benign tumors are misdiagnosed as malignant tumors because of high HE4, the patient's pain and burden will also increase. In this group, the adenomyosis, myoma of the uterus, functional uterine bleeding, and benign adnexal masses that were differentially diagnosed were all subjected to conservative or minimally invasive treatment and showed a satisfactory curative effect.

\section{REFERENCES}

Bandiera E, Romani C, Specchia C, Zanotti L, et al. (2011). Serum human epididymis protein 4 and risk for ovarian malignancy algorithm as new diagnostic and prognostic tools for epithelial ovarian cancer management. Cancer Epidemiol. Biomarkers Prev. 20: 2496-2506.

Bignotti E, Ragnoli M, Zanotti L, Calza S, et al. (2011). Diagnostic and prognostic impact of serum HE4 detection in endometrial carcinoma patients. Br. J. Cancer 104: 1418-1425.

Drapkin R, von Horsten HH, Lin Y, Mok SC, et al. (2005). Human epididymis protein 4 (HE4) is a secreted glycoprotein that is overexpressed by serous and endometrioid ovarian carcinomas. Cancer Res. 65: 2162-2169.

Galgano MT, Hampton GM and Frierson HF Jr. (2006). Comprehensive analysis of HE4 expression in normal and malignant human tissues. Mod. Pathol. 19: 847-853.

Hellström I, Raycraft J, Hayden-Ledbetter M, Ledbetter JA, et al. (2003). The HE4 (WFDC2) protein is a biomarker for ovarian carcinoma. Cancer Res. 63: 3695-3700.

Hertlein L, Stieber P, Kirschenhofer A, Krocker K, et al. (2012). Human epididymis protein 4 (HE4) in benign and malignant diseases. Clin. Chem. Lab. Med. 50: 2181-2188.

Holcomb K, Vucetic Z, Miller MC and Knapp RC (2011). Human epididymis protein 4 offers superior specificity in the differentiation of benign and malignant adnexal masses in premenopausal women. Am. J. Obstet. Gynecol. 205: 358-6.

Jacob F, Meier M, Caduff R, Goldstein D, et al. (2011). No benefit from combining HE4 and CA125 as ovarian tumor markers in a clinical setting. Gynecol. Oncol. 121: 487-491.

Kadija S, Stefanovic A, Jeremic K, Radojevic MM, et al. (2012). The utility of human epididymal protein 4, cancer antigen 125, and risk for malignancy algorithm in ovarian cancer and endometriosis. Int. J. Gynecol. Cancer 22: 238-244.

Kirchhoff C, Habben I, Ivell R and Krull N (1991). A major human epididymis-specific cDNA encodes a protein with sequence homology to extracellular proteinase inhibitors. Biol. Reprod. 45: 350-357.

Lenhard M, Stieber P, Hertlein L, Kirschenhofer A, et al. (2011). The diagnostic accuracy of two human epididymis protein 4 (HE4) testing systems in combination with CA125 in the differential diagnosis of ovarian masses. Clin. 
Chem. Lab. Med. 49: 2081-2088.

Lindquist JA and Mertens PR (2013). Myofibroblasts, regeneration or renal fibrosis-is there a decisive hint? Nephrol. Dial. Transplant. 28: 2678-2681.

Montagnana M, Danese E, Ruzzenente O, Bresciani V, et al. (2011). The ROMA (Risk of Ovarian Malignancy Algorithm) for estimating the risk of epithelial ovarian cancer in women presenting with pelvic mass: is it really useful? Clin. Chem. Lab. Med. 49: 521-525.

Moore RG, Brown AK, Miller MC, Skates S, et al. (2008). The use of multiple novel tumor biomarkers for the detection of ovarian carcinoma in patients with a pelvic mass. Gynecol. Oncol. 108: 402-408.

Moore RG, Miller CM, Brown AK, Robison K, et al. (2011). Utility of tumor marker HE4 to predict depth of myometrial invasion in endometrioid adenocarcinoma of the uterus. Int. J. Gynecol. Cancer 21: 1185-1190.

Moore RG, Miller MC, Eklund EE, Lu KH, et al. (2012a). Serum levels of the ovarian cancer biomarker HE4 are decreased in pregnancy and increase with age. Am. J. Obstet. Gynecol. 206: 349-7.

Moore RG, Miller MC, Steinhoff MM, Skates SJ, et al. (2012b). Serum HE4 levels are less frequently elevated than CA125 in women with benign gynecologic disorders. Am. J. Obstet. Gynecol. 206: 351-358.

Nagy B, Jr., Krasznai ZT, Balla H, Csobán M, et al. (2012). Elevated human epididymis protein 4 concentrations in chronic kidney disease. Ann. Clin. Biochem. 49: 377-380.

Schummer M, Drescher C, Forrest R, Gough S, et al. (2012). Evaluation of ovarian cancer remission markers HE4, MMP7 and Mesothelin by comparison to the established marker CA125. Gynecol. Oncol. 125: 65-69.

Van Gorp T, Cadron I, Despierre E, Daemen A, et al. (2011). HE4 and CA125 as a diagnostic test in ovarian cancer: prospective validation of the Risk of Ovarian Malignancy Algorithm. Br. J. Cancer 104: 863-870. 